\title{
J'AIME MON MUSÉE : LA PERCEPTION ESTHÉTIQUE DES ENFANTS ET LEUR RAPPORT À L'ART
}

Joëlle Lagier, Virginie de Barnier et Kafia Ayadi

\author{
Management Prospective Ed. | « Management \& Avenir »
}

2015/4 N 78 | pages 41 à 57

ISSN 1768-5958

Article disponible en ligne à l'adresse :

https://www.cairn.info/revue-management-et-avenir-2015-4-page-41.htm

Distribution électronique Cairn.info pour Management Prospective Ed..

(C) Management Prospective Ed.. Tous droits réservés pour tous pays.

La reproduction ou représentation de cet article, notamment par photocopie, n'est autorisée que dans les limites des conditions générales d'utilisation du site ou, le cas échéant, des conditions générales de la licence souscrite par votre établissement. Toute autre reproduction ou représentation, en tout ou partie, sous quelque forme et de quelque manière que ce soit, est interdite sauf accord préalable et écrit de l'éditeur, en dehors des cas prévus par la législation en vigueur en France. Il est précisé que son stockage dans une base de données est également interdit. 


\title{
J'aime mon musée : la perception esthétique des enfants et leur rapport à l'art ${ }^{1}$
}

\author{
Joëlle LAGIER ${ }^{2}$ \\ Virginie De BARNIER ${ }^{3}$ \\ Kafia AYADI ${ }^{4}$
}

Résumé

Cet article analyse les perceptions et les attitudes des enfants vis-àvis des musées afin de mieux comprendre leurs attentes. L'objectif de cet article est de comprendre la manière dont les enfants perçoivent la dimension esthétique des musées. Des études qualitatives menées auprès d'enfants âgés de 7 à 12 ans (entretiens semi-directifs) ont permis d'appréhender les différentes approches de ces lieux culturels. Les résultats permettent d'identifier des perceptions et attitudes différenciées selon le degré de familiarité avec ce type de lieu. Les recommandations s'articulent autour d'un axe majeur: développer les aspects ludiques, expérientiels et sensoriels des musées afin que les enfants se sentent acteurs et parties prenantes de cet univers.

Abstract

This paper analyzes children's attitude and perception of museums in order to better understand their expectations towards this place. The aim of this article is to understand how children perceive the

1 Cet article a déjà fait l'objet d'une communication dans le cadre de l'International Marketing Trends Conference - Venise - 24 et 25 janvier 2014.

2 Joëlle LAGIER: Enseignante-chercheuse, Directeur Adjoint du Pôle Marketing ESC La Rochelle. Ses champs de recherche majeurs sont l'esthétique, les arts et la culture lagierj@esc-larochelle.fr

3 Virginie DE BARNIER: Professeur des Universités, Aix-Marseille Université, CERGAM EA 4225, directeur d'Aix-Marseille Graduate School of Management - IAE. Ses recherches au sein du CERGAM se situent dans l'axe Management des Marques et portent sur la communication virginie.de-barnier@iae-aix.com

4 Kafia AYADI : Professeur Assistant au Département Marketing de Neoma Business School, est chercheur dans le domaine de la consommation des enfants - kafia.ayadi@neoma-bs.fr 
aesthetic dimension of museums. Semi-directive interviews were lead with children aged 7-12 years. Results highlighted different perceptions and attitudes vis-à-vis these cultural sites according to children familiarity with them. The recommendations are organized around a major axis: developing fun, experiential and sensorial aspects of museums in order to make children feel as actors and stakeholders of this place.

Si les musées ont pour mission de conserver les œuvres et de les exposer au plus grand nombre, un de leurs engagements est également pédagogique : sensibiliser les enfants à l'art et le rendre plus accessible aux plus jeunes. Or, les musées sont des lieux souvent méconnus, peu fréquentés et peu appréciés des enfants qui préfèrent de loin des lieux plus ludiques, tels que les parcs d'attraction pour lesquels ils constituent une cible marketing claire et identifiée comme telle.

À titre d'exemple, les premiers résultats de l'expérience MuMo, musée itinérant d'art contemporain créé en 2011, indiquent que sur 43000 enfants rencontrés âgés entre 6 et 12 ans, $56 \%$ d'entre eux n'ont encore jamais visité de musées. De même, MerleauPonty (2000), enseignante en muséologie à l'école du Louvre, déplore dans son article intitulé «Les enfants et les musées, encore un petit effort » que tout ce qui touche aux enfants, dans le domaine culturel en particulier, continue à faire l'objet d'un certain mépris. Car les services éducatifs sont souvent les parents pauvres des centres culturels et des musées d'art ainsi que des budgets au sein de l'état. Malgré tout, en France, certains musées proposent aux enfants des ateliers ludiques et créatifs dans un objectif éducatif. Ainsi, le musée d'Orsay propose aux enfants de réaliser des activités de dessin et de peinture ou de moulage. Au musée du Quai Branly, il est possible de fêter son anniversaire au musée avec une heure trente dédiée à des ateliers artistiques et une demi-heure consacrée au goûter au sein du Café Branly. Au musée du Louvre, il existe des parcours et ateliers qui permettent de comprendre une technique artistique.

En 2005, une étude du CREDOC annonce que les moins de 25 ans pensent que les musées n'intéressent pas les enfants car ce sont des lieux ennuyeux et peu attirants (Alibert et alii, 2005). Il est pourtant important que les enfants fassent partie de la réflexion autour de l'art, si l'on souhaite que les musées soient des lieux d'échange culturels pérennes et ouverts à toutes les générations. Une étude de l'INSEE ${ }^{5}$ montre, en effet, que l'attrait pour les loisirs culturels de manière générale (ex : lecture, cinéma, théâtre, visites de musées) qui a été initié durant l'enfance, perdure généralement à l'âge adulte. Ainsi 75 \% des personnes ayant visité 4 ou 5 fois un musée, une exposition ou un monument historique durant leur enfance continuent cette pratique à l'âge adulte. Tandis que seulement $25 \%$ des individus n'ayant pas visité ces lieux durant leur enfance les fréquentent à l'âge adulte. Il est donc essentiel pour les acteurs du monde de l'art de mieux comprendre la perception esthétique que les enfants ont visà-vis de l'art et des musées.

5 INSEE, Les pratiques culturelles: le rôle des habitudes prises dans l'enfance, C. Tavan, $N^{\circ} 883$ - Février 2003. URL: http ://www.insee.fr/fr/ffc/docs_ffc/ip883.pdf 


\section{J'aime mon musée : la perception esthétique des enfants et leur rapport à l'art}

Bien que de nombreuses études aient démontré, dans le domaine du marketing, que les enfants évaluaient les produits principalement selon des éléments d'ordre affectif (Derbaix, 1982 ; Brée, 1993), peu de recherches ont été menées sur le rôle de la dimension esthétique dans le processus perceptuel établi. Selon Machotka (1963), les enfants sont capables de comprendre certaines dimensions esthétiques d'une œuvre ou d'un objet dès l'âge de 5 ans (couleurs, représentativité...). Mc Neal (1992) a démontré que les enfants sont sensibles à la couleur des packagings et établissent une relation entre la nature de l'objet et la couleur de celui-ci. De même, la forme et le conditionnement doivent être originaux et suivre les codes esthétiques attendus (Felix, 1993). Montignaux (2002) a indiqué, de son côté, que la forme et la couleur du packaging pouvaient conduire à une certaine appréciation esthétique des enfants et à un positionnement clair de leur part vis-à-vis de la marque présentée. Dans ce contexte, l'âge (Piaget et Inhalder, 2012 ; Moore, 1973 ; Parsons, 1982), le milieu culturel (Machotka, 1963), le genre et l'implication (Ezan et Lagier, 2009) constituent des caractéristiques importantes dans le choix et les préférences esthétiques développées. Il a été également démontré que certaines couleurs sont associées chez l'enfant à des odeurs (Bellizzi et al., 1983). L'enfant est donc parfaitement à même d'appréhender la dimension esthétique d'objets ou de lieux selon des mécanismes spécifiques. Cependant, si certains constats ont déjà été faits concernant la perception de l'esthétique par les enfants, il reste encore de nombreux champs de recherche peu investigués et ouverts. En particulier, les processus mis en place par les enfants dans l'appréciation esthétique des objets et de leur environnement sont mal connus. De ce fait, il est difficile d'appréhender les attitudes de l'enfant et de comprendre comment ses appréciations finales se forment dans ce contexte.

Cette recherche exploratoire a pour objectif de mieux comprendre comment les enfants évaluent un lieu dont la dimension esthétique est fondamentale : les musées. Afin d'appréhender l'influence de la perception esthétique sur leur perception globale, nous avons choisi d'investiguer le ressenti et le vécu d'enfants vis-à-vis de cet univers particulier. L'objectif est de comprendre les préjugés, heuristiques et attentes des enfants envers les musées. L'analyse a été menée en fonction de leur degré de familiarité avec cet univers, car la familiarité a un impact sur l'attitude, comme l'a montré Zajonc $(1968,1980)$ avec l'effet d'exposition : la répétition d'un stimulus (familiarité) améliore l'attitude envers ce stimulus (agrément). Ainsi la familiarité a un impact sur la formation des croyances, jugements et processus d'évaluation finaux. De même, l'approche de la consommation culturelle selon la dimension expérientielle constitue un angle de recherche intéressant, dans le sens où elle valorise les émotions et les relations affectives engendrées (Holbrook et Hirschmann, 1982).

Nous présenterons, dans une première partie, un état de l'art relatif aux recherches sur la dimension esthétique des musées et son impact sur les attitudes et perceptions des enfants. Nous proposerons, dans une seconde partie, la méthodologie de recherche retenue ainsi que les résultats de l'analyse de la perception et des attitudes des enfants vis-à-vis des musées, en fonction de leur familiarité avec ce contexte. 


\section{La dimension esthétique des musées et son impact sur les attitudes et perceptions des enfants}

La sphère muséale s'est profondément transformée et diversifiée ces trente dernières années, avec notamment une évolution des médiations dans l'exposition (Krebs et Mareska, 2005). Tout le champ culturel s'est ainsi trouvé bouleversé par la révolution numérique, à la fois dans ses contenus, ses usages et ses modes de consommation (Octobre, 2009). Nous assistons dans ce contexte à une hybridation de l'offre muséale en raison d'une porosité croissante entre la sphère culturelle et la sphère des loisirs, plus précisément entre les musées et les parcs d'attraction (Pulh et Mencarelli, 2010). Tentant de s'adapter à de nouvelles attentes des visiteurs, à la recherche d'une expérience muséale plus ludique et plus attractive, cette démarche s'appuie notamment sur des principes et des outils du réenchantement de l'expérience vécue tels que la thématisation, la spatialisation et la scénarisation de l'offre (Ritzer, 2000).

Si cette tendance de la part des musées à rendre leur médiation culturelle plus ludique et interactive pour attirer de nouveaux publics, notamment les jeunes visiteurs, a été réelle et a donné lieu à une mutation des musées en France et à l'étranger, peu de recherches se sont penchées sur l'efficacité de cette modernisation des musées et sur l'impact de ces évolutions sur la perception des musées. Les recherches portant sur l'impact de ces mutations sur la cible que constituent les enfants sont encore moins nombreuses. À notre connaissance, peu de recherches en marketing ont abordé la thématique sous l'angle de la perception des musées par les enfants, seule la sociologie s'est intéressée au phénomène. Nous ne pouvons, en effet, citer ici que l'existence de certaines études sociologiques récentes, dont notamment la recherche menée par Jonchery (2010) sur la représentation des musées par des enfants âgés entre 8 et 11 ans. Cette étude réalisée pour le Ministère de la Culture et de la Communication a délivré à ce sujet un certain nombre de résultats intéressants qui peuvent être synthétisés en quelques points.

Tout d'abord, les associations libres que les enfants rattachent spontanément au terme «musée » se réfèrent majoritairement à des musées d'art, auxquels ils associent des noms («Le Louvre»...), des objets, des sculptures, des tableaux, des peintures (« $L a$ Joconde, le Sphinx»...), mais également des lieux et des endroits où il y a des expositions. La notoriété de la marque muséale ou de l'œuvre est donc un élément fort qui impacte la perception des musées. Cette recherche a également montré qu'il existe différents types de musées, présentant une diversité d'expériences vécues. Si la taille du musée peut constituer un critère typologique, la distinction par catégories thématiques constitue ici la dimension la plus opérante. Ces enfants associent ainsi le musée d'art à « un musée de peintures ou de tableaux », tandis qu'ils rattachent le plus souvent les autres musées à la catégorie des « sciences et techniques » (ex: musée de l'armée de l'air, musée de l'espace, musée d'histoire naturelle...). La typologie des musées opérée spontanément par les enfants est donc directement liée au contenu du musée, opposant art et technique.

La différenciation s'opère aussi selon les médiations mises en place vis-à-vis de l'œuvre et la possibilité ou non que l'enfant se trouve acteur dans l'espace visité. Les enfants interviewés vont, par exemple, indiquer que lorsqu'il y a des guides et des audio-guides, 
il s'agit bien d'un musée. Par ailleurs, ils sont capables de relever que dans un musée les objets ont été déplacés pour être vus, présentés et exposés, contrairement à un monument historique, par exemple, dont la qualité majeure est d'être «in situ».

En outre, le musée fait partie d'un univers culturel complet entouré de châteaux, monuments, zoos, aquariums, planétariums et salons. Les enfants peuvent rencontrer des difficultés pour catégoriser certains de ces événements ou institutions en raison de la proximité de leurs activités avec celles mêmes des musées. Cependant, la plupart de ces enfants pensent que «l'inanimé » est une caractéristique inhérente aux musées.

Les musées reflètent enfin un certain nombre d'activités telles que «raconter, marcher, rêver, regarder, écouter, apprendre, savoir». Le musée est ainsi pour ces enfants à la fois un lieu de promenade enrichissant mais parfois ennuyeux et fatiguant. Les raisons de cette fatigue muséale sont, en fait, diverses. Tout d'abord, la marche est ressentie par les enfants comme une contrainte physique importante qui se prolonge dans le temps. La nécessité d'une contemplation visuelle et d'une écoute auditive passive constitue, par ailleurs, un élément négatif de leur attitude vis-à-vis du musée. Les enfants comparent, en effet, naturellement ces activités à d'autres activités plus attractives et amusantes qui existent dans des institutions éducatives ou culturelles parallèles. Le Palais de la Découverte constitue, par exemple, dans ce contexte un cas de figure, où les enfants apprennent tout en s'amusant et ne voient pas le temps passer.

La possibilité d'apprendre et de savoir grâce au musée reste néanmoins pour ces enfants un élément positif et essentiel, car il est le reflet de certains aspects historiques et culturels de notre civilisation. La visite de musées constitue effectivement, pour eux, un facteur de développement et d'enrichissement, dans le sens où l'acquisition de connaissances est propice à leur croissance et à leur cheminement vers la vie d'adulte, ne serait-ce que pour échanger, communiquer et se positionner dans leur environnement de manière adaptée (de Singly, 1993). L'utilité scolaire est ainsi évoquée, en soulignant l'idée que la visite de musée est un excellent complément d'apprentissage.

Les fonctions et le rôle du savoir au musée restent donc aux yeux de ces enfants des éléments fondamentaux, même s'ils pensent que cela pourrait se passer différemment. Quelques-uns de ces enfants évoquent néanmoins un certain plaisir esthétique et une certaine joie de pouvoir découvrir «de nouvelles choses» dans le cadre de la visite d'un musée.

En conclusion, il semble donc que pour les enfants de 8 à 11 ans, les musées se définissent comme un lieu concret et incontournable, où il $\mathrm{y}$ a rencontre et confrontation avec l'objet, où se développent des activités culturelles directement liées à la culture et au savoir. Cette rencontre est également source de certaines attitudes négatives, en raison de l'effort physique demandé et de la difficile participation interactive à la démarche menée.

Ces résultats ne sont pas sans faire écho aux transformations du paysage muséal depuis les années 80 , mais ne présentent pas pour autant de conclusions très encourageantes, en ce qui concerne l'approche muséale des enfants. Car si ces derniers reconnaissent la nécessité et l'intérêt de visiter un musée pour apprendre et se cultiver, ils ne semblent pas être totalement convaincus et satisfaits de l'offre et 
des services proposés, malgré l'ensemble des actions menées à ce sujet. Or, si nous revenons, dans ce contexte à l'apprentissage et la perception esthétique de l'enfant, nous pouvons constater que l'enfant n'appréhende pas son environnement de la même manière qu'un adulte. Ses développements cognitif et affectif sont distincts.

En effet, selon Piaget (1989), la pensée de l'enfant possède une structure originale spécifique. L'auteur distingue quatre stades perceptuels : le stade «sensori-moteur» (enfants de moins de deux ans), le stade «préopératoire» (enfants de 2 à 7-8 ans), le stade « opératoire concret » (enfants de 7-8 ans à 11-12 ans) et enfin, le stade « opératoire formel » (après 11-12 ans). Lorsque l'âge et les stades se développent, la mémoire ainsi que la capacité à exécuter des tâches cognitives plus complexes se développent également.

Parallèlement aux travaux de Piaget (1989), des recherches ont été menées en psychologie sur le développement de la sensibilité esthétique de l'enfant. Une étude assez ancienne mais très intéressante sur la perception esthétique des enfants concernant les tableaux (peintures occidentales depuis la Renaissance) a mis en en évidence trois stades de développement des critères esthétiques (Machotka, 1963) :

- le premier stade commence avant l'âge scolaire et se termine vers 7 ans. La couleur est le premier élément qu'apprécient les enfants bien avant le sujet représenté ;

- le second stade débute vers l'âge de 7-8 ans et se poursuit jusque douze ans. Durant cette période, l'enfant insiste sur le réalisme du tableau et considère le contraste, l'harmonie et la clarté de l'œuvre ;

- $\quad$ le troisième stade débute vers l'âge de douze ans. L'adolescent présente moins d'intérêt pour la couleur et le réalisme du tableau. Il va plutôt évaluer l'œuvre présentée selon son style, sa composition, sa luminosité et son contenu affectif (correspondant à la fois à l'identification qu'il peut faire avec sa propre histoire ou à la nature même de l'œuvre).

Ainsi, tant sur le développement intellectuel de l'enfant que sur sa sensibilité aux critères esthétiques, la tranche d'âge de 7-12 ans apparaît comme étant la plus appropriée dans l'étude du comportement de l'enfant (Brée, 1993). Avant sept ans, l'enfant n'intègre effectivement pas assez d'éléments dans l'analyse des stimuli esthétiques présentés, trop focalisé sur les aspects plus ludiques de ses activités. À partir de sept ans, il est capable de développer un certain réalisme visuel et peut établir la comparaison entre ce qu'il voit et la représentation réelle. Après 12 ans, la pensée de l'adolescent se rapproche davantage de celle de l'adulte.

Il nous a donc semblé intéressant d'approfondir et de poursuivre ces travaux antérieurs (Jonchery, 2010), en prenant à nouveau le point de vue de l'enfant vis-à-vis des musées mais en essayant de comprendre de manière plus approfondie et plus spécifique les perceptions et les attitudes des enfants âgés entre 7 et 12 ans. Notre approche a privilégié l'étude du ressenti des enfants vis-à-vis des musées par la conduite d'études qualitatives opposant enfants familiers des musées et enfants peu familiers. La méthodologie est explicitée précisément dans la partie suivante. 


\section{Les perceptions et attitudes des enfants vis-à-vis des musées en fonction de leur familiarité}

\subsection{La méthodologie}

En nous appuyant sur un guide d'entretien élaboré à cet effet, nous avons mené 16 entretiens semi-directifs individuels auprès d'enfants âgés de 7 à 12 ans avec une répartition égalitaire des sexes. Ces enfants étaient originaires des villes de Rouen, Caen ou de leurs alentours et présentaient des caractéristiques différentes en termes de sensibilisation à l'art. Tandis que 10 d'entre eux n'ont, en effet, jamais visité de musée ou en ont visité un lors d'une sortie avec l'école, les 6 autres ont déjà fait plusieurs visites de musées en famille ou avec l'école. Privilégiant la parole de l'enfant, l'entretien a été mené sans la présence des parents et organisé en plusieurs étapes, afin de soutenir la concentration des enfants et permettre le recueil de différents matériaux. Les entretiens ont été entièrement enregistrés et les verbatim retranscrits pour l'analyse des données qualitatives.

Les enfants peu familiers des musées se sont ainsi exprimés librement sur différents thèmes : l'identité d'un musée (qu'est-ce qu'un musée ?), les appréciations spontanées positives et négatives vis-à-vis des musées, les suggestions de choses intéressantes à faire dans un musée... Le guide d'entretien destiné aux enfants familiers des musées comprenait les mêmes questions, mais il leur était également demandé de citer les musées qu'ils avaient appréciés ainsi ceux qu'ils n'avaient pas aimés et de justifier pourquoi. Enfin, les enfants familiers aux musées ont été interrogés à la sortie même d'une visite de musée et il leur a été demandé de raconter cette dernière expérience, en mettant en lumière ce qu'ils avaient ressenti de positif et de négatif dans ce contexte précis.

\subsection{Les résultats obtenus}

Les résultats ont été mis en perspective grâce à une analyse thématique qui permet de mettre en exergue les thèmes majeurs évoqués spontanément par les enfants et de différencier les thématiques en fonction de la familiarité ou non des enfants avec les musées. L'analyse a été réalisée séparément par deux chercheurs, les résultats confrontés et en cas de désaccord un troisième chercheur a arbitré afin que les thématiques principales soient parfaitement identifiées.

Les résultats sont présentés par groupe d'enfants (peu familier Vs familier) et par thématique.

\subsubsection{Les perceptions et attitudes des enfants peu familiers avec les musées}

Quatre thématiques majeures ressortent de l'analyse des entretiens : le caractère inaccessible des musées, l'absence de ludique et de sensoriel, le manque d'une approche expérientielle et personnelle et enfin, l'intérêt mais dans un horizon temporel très lointain. Ces thématiques sont détaillées ci-après. 


\section{Un lieu abstrait et immatériel}

Dans leur appropriation du musée, les enfants peu familiers avec les musées décrivent, dans leur majorité, le musée comme un lieu abstrait et immatériel, sans finalité ou inutile : «moi, je ne sais pas trop à quoi ça sert» (M., 8 ans). Un petit nombre d'entre eux (deux) a, au contraire, idéalisé le lieu comme L. 11 ans : "le musée, c'est un grand palais avec les murs dorés et des grande pièces [...] ça coûte très cher pour y rentrer, normal vu que c'est quand même du luxe».

Interrogés aussi sur la cible des musées, les enfants interviewés ne se sont pas identifiés comme une cible potentielle de ce lieu. Les qualificatifs donnés par ces derniers sur les caractéristiques de la cible visée ont, en effet, été les suivants : pour les «intellos», «les gens cultivés », «les vieux » ou encore «les riches ».

Même si les principales sources d'informations sur les musées proviennent de l'école ou de la télévision, notamment les dessins animés et certaines émissions pour enfants, la définition d'un musée et de son environnement demeure donc encore pour ces enfants floue et vague, représentant, de toutes les façons, un lieu inaccessible en raison principalement du niveau intellectuel et du coût exigé. Un enfant nous a cité néanmoins un nom d'artiste: «dans les musées, tu trouves plein de choses rares comme des tableaux de, heu, Picasso. Tu sais c'est celui qui fait des peintures un peu bizarres avec des têtes déformées » (L., 11 ans). La notoriété de l'œuvre, de l'artiste ou du musée a donc une fonction forte dans la représentation des musées par les enfants peu familiers avec les musées.

\section{L'absence de ludique et de sensoriel}

Les enfants peu familiers avec les musées se sont également exprimés sur l'appréciation du musée ou de ce qu'il représente à leurs yeux. Les réactions ont été ici de deux ordres. Sept enfants ont eu un comportement de rejet comme l'illustre le verbatim suivant: «moi, je n'aimerais pas aller au musée parce que c'est ennuyeux, c'est super long et silencieux. On ne peut pas s'amuser comme quand on va à la piscine ou au parc » (R. 10 ans). Pour ces enfants, le musée n'évoque ni le plaisir ni la stimulation. L'absence de ludique et de sensoriel les gêne profondément : «j'aime bien aller au parc Astérix, mais au musée ça ne m'intéresse pas. Déjà que je travaille à l'école, je n'ai pas envie de réfléchir encore!» (P. 10 ans). Les trois autres enfants ont cependant évoqué une image positive des musées et pensent même demander à leurs parents de les y emmener.

\section{La demande d'une approche expérientielle et personnelle}

Les attentes concernant le musée portent davantage sur l'atmosphère que sur le contenu lui-même. Dans ce contexte, les enfants semblent, en effet, plus sensibilisés par l'environnement sensoriel que les œuvres proprement dites du musée.

Les plus jeunes nous ont ainsi fait part de leurs attentes en matière d'activités divertissantes lors de la visite des musées : «ce serait bien si on pouvait avoir des toboggans et tout ça à l'extérieur, comme ça on peut s'amuser et rigoler avec les copains » (M. 8 ans). 
Par contre, les enfants plus âgés ont imaginé un musée qui serait le reflet de leurs centres d'intérêts : «dans un musée idéal, j'aimerais bien voir des voitures de collection. Mes préférées à moi c'est les Ferrari ! Je les collectionne. Les miniatures bien sûr ! (rire)» (Y. 11 ans).

\section{Le paradoxe : j'irai bien quand je serai grand...}

Sur le plan conatif, il a été également observé un certain paradoxe. En effet, les enfants, qui ont rejeté le musée, ont néanmoins manifesté de l'intérêt pour visiter ce lieu.

Il est important de souligner cependant que la fréquentation éventuelle d'un musée peut s'envisager dans l'avenir à l'âge adulte : «ça serait bien quand même d'y aller plus tard quand je serai un peu plus grand parce que quand même, ça doit être intéressant et comme ça je connaîtrai plus de choses » (F. 9 ans).

\subsubsection{Les perceptions et attitudes des enfants familiers avec les musées}

De nouveau quatre thématiques majeures ressortent de l'analyse des entretiens : le caractère unique, féérique, exceptionnel, l'offre plurielle, la référence au passé et l'aspect esthétique, participatif et sensoriel des musées. Ces thématiques sont détaillées ci-après.

\section{L'unique, le féérique, l'exceptionnel}

Pour les enfants plus experts, le musée représente, de son côté, un univers unique et exceptionnel : " on y voit des choses que l'on ne peut pas voir ailleurs [...] par exemple des tableaux uniques [...] il en existe qu'un comme celui-là» (A. 7 ans).

Le musée constitue, pour eux, un lieu original : «c'est un endroit où l'on peut observer les choses qui sont un peu bizarres, comme un naufrage, des statues... » (T. 7 ans). Ce lieu est représenté la plupart du temps par un bâtiment, un édifice de grande taille : «le musée pour moi, c'est comme un château, il y a des fourchettes, des couteaux, des meubles que les rois et la reine utilisaient autrefois » (M. 8 ans) ; «Un musée, c'est comme un immeuble parce qu'ily a beaucoup de fenêtres [...]. C'est grand» (F. 7 ans). Dans ce même contexte, cet endroit peut prendre parfois des dimensions magiques, voire féériques : «regarde ce tableau, on dirait qu'il y a une princesse dedans » (M. 8 ans).

\section{L'offre plurielle}

Contrairement à l'enquête menée précédemment par Jonchery (2010) pour le Ministère de la Culture et de la Communication, les enfants ici interrogés n'associent pas obligatoirement une catégorie d'objets à un certain type de musée.

Pour la grande majorité, l'objet du musée peut être diversifié, sachant que cet objet doit être toujours unique et original : «c'est un endroit où l'on peut voir 36000 trucs, des œuvres, des tableaux mais aussi des objets... » (R.10 ans) ; «c'est un lieu où on ne voit pas que des tableaux, un lieu où on voit des choses différentes, des choses normales, des choses pas normales [...] par exemple, des peintures, on en fait encore dans les musées... » (M. 8 ans). 


\section{L'ancien, la référence à l'Histoire, au passé}

L’objet exposé au musée doit être également ancien ou faire référence au passé : « $a u$ musée, tu vois des choses anciennes que l'on ne veut pas voir dans une maison moderne» (A. 7ans) ; «on y voit des objets qui sont très vieux, d'il y a longtemps » (M. 8 ans).

Cette approche peut ainsi faire référence à des métiers d'autrefois : «dans un musée, on voit des choses que l'on utilisait avant pour vivre comme par exemple des meubles, des métiers, le métier du maréchal-ferrant, l'atelier du cordonnier» (F. 7 ans), et permettre une certaine reconstruction du passé : "j'ai vu dans ce musée comment la ville était avant la guerre [...] c'était pas du tout pareil et ça c'est vraiment intéressant parce que tu vois ce qui a changé... » (A. 7 ans) ; «j'ai bien aimé ce que la dame nous a expliqué sur le tableau de Granville. On n'allait pas se baigner, il y avait pas des tentes, il y avait des cabines roulantes sur la plage. C'est intéressant parce qu'on ne savait pas » (M. 8 ans).

\section{L'aspect esthétique, participatif et sensoriel}

Les enfants familiers avec les musées expriment aussi clairement un certain plaisir esthétique ou sensoriel : « un endroit où l'on expose de belles choses... » (A. 7 ans) ; «un musée, c'est gai » (R. 10 ans) ; " une visite de musée, c'est drôle... c'est grand... en plus, il faut monter des escaliers... » (T. 7 ans). Mais ce plaisir est vécu différemment selon les types de sensibilités rencontrés.

Pour un enfant, ce plaisir se développe, en effet, dans un lieu, où la sérénité et la tranquillité sont appréciées : « un lieu calme... enfin, on entend les gens qui bavardent mais on entend pas les gens qui crient... » (A. 7 ans), tandis que pour les cinq autres, il s'agit de pouvoir s'exprimer physiquement ou verbalement lors de la visite d'un musée : "c'est bien parce qu'elle (la guide) n'a pas que parlé [...] elle nous a posé des questions, sinon c'est trop ennuyeux [...] elle nous a demandé notre avis. Je me rappelle qu'elle a demandé si la mer est agitée ou pas... Est-ce qu'il pleut, est ce qui pleut pas... quel objet on voit... » (M. 8 ans); "les expériences qui étaient bien, c'est que l'on faisait des choses à l'atelier en mélangeant des choses qui faisait un truc gazeux comme le coca [...] moi, j'aime bien les expériences [...] j'ai mieux aimé que l'atelier peinture [...] À l'école aussi, on fait des expériences, on mélange par exemple du jaune et du vert» (F. 7 ans).

Cette activité recherchée se traduit par un intérêt encore plus prononcé de l'enfant, lorsque l'expérience vécue est en relation directe avec ce qui a été vu au musée, à l'école ou fait à la maison : "j'ai peint un arbre à l'aquarelle, c'était bien [...] j'ai peint quelque chose de l'extérieur, un arbre comme dans des tableaux que l'on a vus au musée aujourd'hui » (M. 8 ans) ; "aujourd'hui, j'ai peint à l'aquarelle et je pense que certains tableaux que l'on a vus avant étaient aussi peints à l'aquarelle [...] C'était bien parce qu'à la maison, j'ai que des pots de peinture à l'eau... » (A. 7 ans).

Le souvenir, dans tous les cas, avec une histoire antérieure est ressenti de manière très positive et engendre une certaine forme de satisfaction : "par exemple, le tableau avec une tempête près d'une falaise, c'est mon tableau préféré [...] parce que j'ai déjà fait ça, j'ai déjà eu une tempête en prenant le bateau» (M. 8 ans) ; "j'ai bien aimé cette visite de musée parce que c'était la mer et le thème à mon école, c'est la mer» (F. 7 ans). 
Le musée ne constitue pas pour autant pour ce second groupe d'enfants plus avertis un pur lieu de divertissement: «un musée, ce n'est pas une récréation [...] y a pas de balançoire par exemple et de toutes façons, ce ne serait pas l'endroit idéal pour les balançoires » (A. 7 ans) ; «non un musée, ce n'est pas un endroit pour s'amuser [...] c'est un endroit où il y a des choses bizarres» (M. 8 ans).

Dans ce contexte, deux enfants émettent des vœux plus spécifiques, reflétant la recherche de certaines sensations complémentaires : «ce serait bien qu'au musée, il y ait de la musique aussi [...] par contre, des odeurs non» (A. 7 ans) ; «j'aimerais bien peindre dans un musée un tableau du musée, mon tableau préféré, à côté du tableau, sur un chevalet» (F. 7 ans).

\section{L'évocation d'un musée idéal pour les enfants quel que soit leur degré de fami- liarité vis-à-vis des musées}

Interrogés sur l'idée d'un musée idéal, tous les enfants évoquent la nécessité de mettre en place des activités diverses et variées pour éviter la lassitude ou la monotonie dans ce contexte : «il faut faire des choses différentes d'un musée à l'autre, car sinon on s'ennuie» (A. 7 ans).

Cet ennui se manifeste, comme dans la première étude, par un temps trop long et une fatigue déclenchée, le plus souvent, par la position debout: «mais des fois au musée, on s'ennuie parce que c'est trop long... Maintenant, j'ai envie de rentrer chez moi [...] je n'ai pas envie de retourner au musée» (R. 10 ans) ; «c'est un peu long [...] on est obligé de rester debout et moi j'aime pas trop. On marche beaucoup》 (F. 7 ans); «c'est bien quand on s'assoit» (M. 8 ans).

Les enfants évoquent enfin, à ce sujet, des idées amusantes et créatives, le plus fréquemment en rapport avec leur propre goût ou centre d'intérêt: «je ferais mon musée dans un endroit ancien avec des vieilles pierres [...] pas trop de plâtre» (A. 7 ans).; «ce serait quelque chose de grand, de carré avec des objets vieux de la guerre [...] des morceaux d'avion par exemple [...] j'ai vu ça à la télé [...] c'était des morceaux d'avion que l'on avait retrouvés dans la terre» (M. 8 ans) ; «je construirais un musée un peu bizarre [...] à l'intérieur et à l'extérieur » (T. 7 ans) ; «je ferais une maison avec plein de fenêtres, avec des murs, des tableaux, et j'aimerais m'y balader avec d'autres enfants... » (R.10 ans).

\section{Conclusion, implications et recommandations}

Les résultats de notre recherche montrent que la perception et l'attitude que les enfants ont des musées sont différentes selon leur degré de familiarité avec ce type de lieu. Il apparaît, en effet, que les enfants non sensibilisés à l'art sont peu intéressés par les musées. Ils ont des préjugés à connotation plutôt négative vis-à-vis de cet environnement et mettent en place des heuristiques de représentativité (Tversky et Kahneman, 1974; Pham, 1996) du type : le musée contient des œuvres chères, donc le musée est cher. Ainsi pour ces enfants peu familiers avec les musées, le musée relève du domaine de l'inaccessible, voire du luxe. Une image caricaturale muséale semble s'être formée dans leur esprit : un lieu ennuyeux, triste, silencieux, fatiguant. Le musée est un monde pour les adultes, un univers qui leur est étranger et qui leur fait un peu peur. Dès lors que 
les enfants sont déjà allés dans un musée, l'image devient positive : le musée relève de l'inédit, de l'original, de l'unique que l'on a grand plaisir à découvrir. Les enfants plus avertis les reconnaissent comme des lieux merveilleux, reflet d'une certaine époque riche en histoire et fortement ancrée dans le passé.

Qu'ils soient familiers ou non avec l'univers des musées, il apparaît que les musées subissent en général, une image peu conforme à la réalité, soit exagérément négative, soit à l'inverse exagérément positive.

Il semble, de ce fait, important que les musées, subissant parfois une image décalée, mettent en place des actions constructives et valorisantes vis-à-vis de toutes les catégories d'enfants. Au vue de l'étude menée en amont et des résultats de notre étude qualitative, l'attractivité des musées doit être, en effet, revue et travaillée de manière différenciée selon les cibles visées.

La théorie du comportement décisionnel sur laquelle la recherche sur les heuristiques se fonde permet de mieux anticiper les comportements des consommateurs et leur prise de décision. C'est au niveau de la formation des croyances de ces heuristiques qu'il est important d'agir afin de réajuster l'image du musée à sa réalité.

Pour les enfants peu familiers avec les musées, il serait, par exemple, judicieux de mettre en place des dispositifs plus ludiques et plus expérientiels, qui leur permettent d'apprendre tout en s'amusant. Ces enfants souffrent effectivement d'une image abstraite, immatérielle des musées et leur attribuent un côté passif voire désuet, en indiquant malheureusement que ce sera un lieu où ils iront peut-être lorsqu'ils seront plus grands. Pour éviter cet écueil et redynamiser leur vision, il serait intéressant de concevoir, au sein des musées, des espaces de co et récréation, où tous les sens des enfants seraient mis en éveil en relation directe avec le lieu, le discours et le contenu des œuvres.

Il a, en effet, été démontré récemment que chez les enfants les effets des visites « actives et multi-sensorielles » sont bénéfiques et supérieurs à ceux de visites «classiques académiques » (Gentaz, Lagier, Pinchon, 2012). Ces visites « actives et multi-sensorielles » s'appuient sur deux principes complémentaires : un engagement actif et une attention pilotée. L'apprentissage est effectivement plus efficace lorsque l'enfant, sollicité par un exercice ou un jeu, essaie de générer lui-même une réponse.

Ces nouveaux univers pourraient donc être conçus, par exemple, via des parcours de découverte olfactifs, tactiles, gustatifs, sonores et visuels élaborés autour de thématiques telles que l'histoire du musée, son design, son architecture ou encore la nature, la composition des œuvres exposées (les supports, les types de medium utilisés, les teintes, les couleurs présentées...).

Cette approche permettrait de rattacher le musée de manière ludique à la vie quotidienne de l'enfant et à ses centres d'intérêt en lui posant des questions telles que: "peux-tu nous raconter à quoi cette couleur, cette texture te font penser ? ", " ce tableau te rappelle-t-il un souvenir, une musique, une odeur particulière ? Si oui, lesquels ?...». Dans ce cadre, des ateliers expérimentaux pourraient être mis en place afin que les enfants expriment librement leurs ressentis, leurs sensations dans l'environnement spécifique que représente un musée. 


\section{J'aime mon musée : la perception esthétique des enfants et leur rapport à l'art}

Ce genre d'expérience originale permettrait d'ancrer la perception que l'enfant a d'un musée dans un univers plus concret par le biais d'expériences vécues de manière pratique et réelle. La dimension relationnelle établie deviendrait, de ce fait, plus affective et donc plus positive.

Le concept d'éduvertissemment (Addis, 2005), centré sur l'association de l'éducation et du divertissement, porte déjà ses fruits dans de nombreuses institutions culturelles et muséales internationales. Mais là où le ludique semble parfois jouer un rôle prédominant, au détriment d'une certaine approche artistique, nous préconisons ici une nouvelle démarche muséale, dans lesquelles les œuvres restent le cœur central des activités et des événements pour le plus grand plaisir des enfants. Il faut éviter, en effet, dans ce contexte, le risque d'une trop grande "dysneylandisation des musées » (Ballofet, Courvoisier et Lagier, 2013), phénomène qui débouche le plus souvent sur un curieux amalgame (King, 1993) entre musées et parcs d'attraction.

L'école, où l'enfant apprend, peut aussi jouer dans ce contexte un rôle très important. Il s'avère effectivement que les sorties pédagogiques dans les milieux culturels sont prescrites par l'Education Nationale au nom d'une égalité culturelle pour tous les élèves. Mais parfois, cette sortie cultuelle n'est pas accompagnée de projet particulier. Il faut donc privilégier les sorties culturelles avec projet à l'appui, pour un réel apprentissage qui fera le lien entre le travail effectué en classe, et celui effectué au musée (Musy, 2011).

Pour les écoles au budget plus limité ne pouvant effectuer ce type de sortie, il semble que les ressources documentaires puissent constituer aussi un très bon support d'enseignement. Par exemple, le site d'un musée comme le Louvre peut être un outil pédagogique très utile pour une visite virtuelle. L'utilisation des TICE en milieu scolaire demande cependant un investissement au niveau du matériel. Toutes les écoles ne possèdent malheureusement pas de salles informatiques. De plus, il faut former les personnels enseignants à la bonne utilisation de ces technologies, et cela aussi coûte de l'argent.

C'est pourquoi nous recommandons ici, en complément, le partenariat des écoles avec des structures extérieures idéalement bénévoles, afin que l'enseignant puisse établir des liens pour préparer le savoir à transmettre. Dans ce contexte, nous considérons que le musée n'est pas un prolongement de l'école mais un complément. Les collections des musées sont, en effet, le plus souvent en accord avec les demandes des programmes scolaires pour la connaissance des œuvres de base. Ainsi école et musée se complètent, en apportant réciproquement des approches différentes. Dans un musée, l'enfant a une approche immédiate de l'œuvre. À l'école, il peut bénéficier d'outils en amont qui lui permettent de mieux l'appréhender.

À ce sujet, les enfants plus familiers aux musées évoquent des impressions beaucoup plus positives vis-à-vis des œuvres et des musées, liés pour eux au domaine de l'exceptionnel, du magique, voire du féérique.

Cette relation avec l'environnement muséal va parfois si loin qu'ils en viennent à imaginer un musée idéal, où ils pourraient venir peindre aux cotés des œuvres présentées ou encore y apporter leurs propres collections... Dans cette même lignée de la rencontre entre l'école et le musée, nous pourrions imaginer ici la création d'un mini musée à 
l'école ou d'un musée idéal au sein du musée même, à l'image de ce qu'ils souhaiteraient faire, voir, créer ou déposer dans ce lieu particulier.

Il existe effectivement une demande commune d'évasion et de divertissement de la part de tous les enfants interrogés, centrée sur une volonté de participation dynamique et interactive au sein du lieu visité, même si le musée doit garder son identité majeure, qui est celle de transmettre de la connaissance et du savoir.

Ces éléments peuvent devenir des axes de communication forts auprès des enfants afin de revaloriser l'image institutionnelle des musées en soulignant la dimension expérientielle de ce type de consommation culturelle (Holbrook et Hirschmann, 1982).

En conclusion, il semble donc que dans le domaine des musées, l'éducation expérientielle et ludique constitue une dimension fondamentale à exploiter auprès des enfants, car cela leur permet d'apprendre en se distrayant et de se distraire en apprenant. Si nous ne souhaitons pas faire de l'enfant «un braconnier des politiques culturelles » (Sirota, 1998a), il faut, de toutes les façons, prendre en compte le point de vue de l'enfant, un enfant considéré comme acteur social et non plus récepteur passif. Car comme l'indique à nouveau Sirota (1998b, p.21) : «Les enfants doivent être considérés comme des acteurs à part entière et non pas simplement des êtres en devenir. Les enfants sont à la fois produits et acteurs des processus sociaux. Il s'agit de renverser la proposition classique, non pas de s'interroger sur ce que fabriquent l'école, la famille ou l'Etat mais de se demander ce que fabrique l'enfant à l'intersection de ces instances de socialisation ».

Nous avons néanmoins conscience, dans ce cadre, de la limite de cette recherche qui revêt un caractère exploratoire. C'est la raison pour laquelle nous souhaitons par la suite ouvrir de nouvelles voies d'investigation, en complétant nos entretiens et en nous appuyant sur les registres psychologiques, heuristiques et sociologiques de l'enfant, afin de mieux appréhender encore le sujet et mieux comprendre cette cible spécifique que constituent les enfants. Il est, en effet, essentiel de savoir comment s'adresser à cette jeune cible afin de s'assurer d'une diffusion de la culture au plus grand nombre.

Plusieurs voies de recherche restent ouvertes. Sur le plan conceptuel, notre recherche montre que les enfants peuvent être familiers avec les musées et pourtant ne pas les aimer. Le lien entre «nombre d'expositions » et « agrément» ne semble donc pas fonctionner sur la cible enfantine. Pourtant, Zajonc (1968) a démontré l'effet d'exposition, le «mere exposure effect», stipulant que la multiplication des expositions à un stimulus engendrait une meilleure attitude envers ce stimulus. Il serait ainsi pertinent de multiplier les travaux afin de comprendre pourquoi nous n'avons pas trouvé les effets attendus par l'effet d'exposition. Il s'agirait ici de centrer la recherche sur la répétition de l'exposition, afin de mesurer à partir de combien de répétitions l'attitude des enfants envers les musées s'améliore. Sur le plan plus managérial, il serait intéressant d'élargir notre recherche à d'autres catégories d'âge, en particulier, les enfants de 2 à 7 ans appartenant au stade préopératoire, et ceux de plus de 11 ans appartenant au stade opératoire formel. Ainsi, les recommandations d'amélioration de l'offre muséale pourraient être différentes en fonction de la tranche d'âge d'enfant visée.

Les responsables de musées doivent également apprendre à combiner les nouvelles technologies dont les jeunes sont férus, aux héritages culturels qu'ils souhaitent trans- 
mettre. L'ouverture et la culture des jeunes générations est, en effet, un défi qu'il est important de relever si l'on tient à ce que la culture reste un élément clé de notre société. Dans ce défi, seuls ceux qui sauront allier l'ancien et le nouveau, l'héritage et la technologie, le culturel et le ludique sauront attirer l'attention et l'intérêt des jeunes générations.

\section{Bibliographie}

ADDIS M. (2005), "New Technologies and Cultural Consumption - Edutainment Is Born!”, European Journal of Marketing, Vol. 39, No. 7-8, p. 729-736.

ALIBERT D., BIGOT R., HATCHUEL G. (2005), « Fréquentation et image des musées au début $2005 »$, Collection de Rapports $\mathrm{N}^{\circ} 240$.

BALLOFET P., COURVOISIER F. \& LAGIER J. (2013 ), "From museum at the amusement park : opportunities and risks of eduvertissement", International Journal of Arts Management, Vol. 16, No 2, p.4-18.

BANDURA A. (1977), Social Learning Theory, Englewood cliffs, NJ : Prentice - Hall.

BELK R.-W., MAYER R. et DRISCOLL A. (1984), “Children's Recognition of Consumption Symbolism in Children's Products", Journal of Consumer Research, No.10, p. 386-397.

BELLIZZI J.-A., CROWLEY A.-E, HASTY R.-W. (1983), "The effects of color in store design", Journal of Retailing, Vol. 59, No.1, p. 21-45.

BREE J. (1993), Les enfants, la consommation et le marketing, Presses Universitaires de France, Paris.

BOURDIEUX P. (1979), La Distinction - Critique sociale du jugement, édition Minuit.

DERBAIX C. (1982), «L'enfant, la consommation publicitaire et la hiérarchie des effets », Revue Française du Marketing, No. 58, p. 7-26, septembre-octobre.

EZAN E. \& LAGIER J. (2009), "How do children develop their aesthetic sensibility", Young Consumers, Vol. 10, No.3, p. 238-247.

FÉLIX M. (1993), «Communication non verbale et Stratégie Produit: Une proposition de modèle de choix au point de vente. Une application aux enfants », Thèse de Doctorat en Sciences de Gestion, Université de Lille 1.

GENTAZ E., LAGIER V. \& PINCHON C. (2012), « Comment favoriser l'acquisition de connaissances artistiques par des enfants et leurs intérêts durant une visite guidée à un musée de peinture? », Culture \& Musées: Revue Internationale de Muséologie et de recherches sur la Culture, No.19, p.171-178.

HOLBROOK M.-B. et HIRSCHMAN E.-C. (1982), "The Experiential Aspects of Consumption : Consumer Fantasies, Feelings, and Fun", Journal of Consumer Research, No. 9, p.132-140.

JONCHERY A. (2010), «Explique-moi le musée, dessine-moi un musée, une analyse des représentations des musées chez les 8-11 ans», Actes du colloque international, Ministère de la Culture et de la Communication, Université Paris Descartes, $9^{\text {èmes }}$ Journées de sociologie de l'enfance, Paris. 
KING M.-J. (1993), The American Theme Park: A Curious Amalgam In Continuities in Popular Culture: The Present in the Past and the Past in the Present and Future, R.B. Browne and R.J. Ambrosetti eds., p. 49-60, Bowling Green, OH : Bowling Green State University Popular Press.

KREBS A. et MARESKA B. (2005), Le renouveau des Musées, La Documentation Française, Collection «Problèmes Politiques et Sociaux», Paris.

MC NEAL J.-U. (1992), Kids as Customers, Lexington Books.

MACHOTKA P. (1963), «Le développement des critères esthétiques chez l'enfant », Enfance, Vol.16, n4-5, p. 357-379.

MERLEAU PONTY C. (2000), La lettre de l'OCIM, $\mathrm{n}^{\circ} 72$.

MIRGALET C. (1997), «Comment le goût esthétique vient aux enfants », Tréma [En ligne], 11 | mis en ligne le 28 novembre 2010, URL, http ://trema.revues.org/1945

MONTIGNEAUX N. (2002), Les marques parlent aux enfants grâce aux personnages imaginaires, Editions d'Organisation, Paris.

MOORE b.-E. (1973), “A Description of Chidren's Verbal Responses to Works of Art in Selected grades", Studies in Art Education, Vol. 14, No. 3, p.27-34.

MUSY A. (2011), «Comment les enfants perçoivent-ils l'œuvre d'art? », Mémoire IUFM Nord Pas de Calais, Université d'Artois.

OCTOBRE S. (2009), «Pratiques Culturelles chez les jeunes et institutions de transmission : un choc de culture?», Culture Prospective, No.1.

PARSONS M.-J. (1976), "A Suggestion Concerning the Aesthetic Development of Children", Journal or Aesthetics and Art Criticism, Vol. 14, No. 3, p. 305-314.

PIAGET J. et INHELDER B. (2012), La psychologie de l'enfant, Presses Universitaires de France, Paris.

PHAM M.-T. (1996), « Heuristiques et biais décisionnels en marketing», Recherches et Applications en Marketing, Vol.11, No. 4, p. 53-69.

PULH M. et MENCARELLI R. (2010), « Muséo-parcs et réenchantement de l'expérience muséale : le cas de la Cité des Arts et des Sciences de Valencia », Décisions Marketing, No. 60, p. 21-31.

RITZER G. (2000), The McDonaldization of Society, An Investigation into the Changing Character of Contemporary Social Life, 3rd ed., Thousand Oaks (CA) : Pine Forge Press.

DE SINGLY F. (1993), Sociologie de la famille contemporaine, Nathan, Paris.

SIROTA R. (1998a), « Nouvelles sociabilités enfantines », Informations Sociales, No.65, p.104-111.

SIROTA R. (1998 b), « L'émergence d'une sociologie de l'enfance : évolution de l'objet, évolution du regard », Education et Sociétés, n² 2 , pp 9-33.

TVERSKY A. et KAHNEMAN D. (1974), "Judgment Under Uncertaint : Heuristics and Biaises", Science, No. 185, p. 1124-1131. 
J'aime mon musée : la perception esthétique des enfants et leur rapport à l'art

WARD S., WACKMAN D.-R. et WARTELLA E. (1977), How children learn to buy: the development of consumer information processing skills, Beverly Hills, Sage Publications.

ZAJONC R.-B. (1968), "Attitudinal Effects of Mere exposure", Journal of Personality and Social Psychology, Vol. 9, N2, p. 1-27.

ZAJONC R.-B. (1980), "Feeling and thinking: Preferences need no inferences", American Psychologist, Vol.35, No 2, p.151-175. 